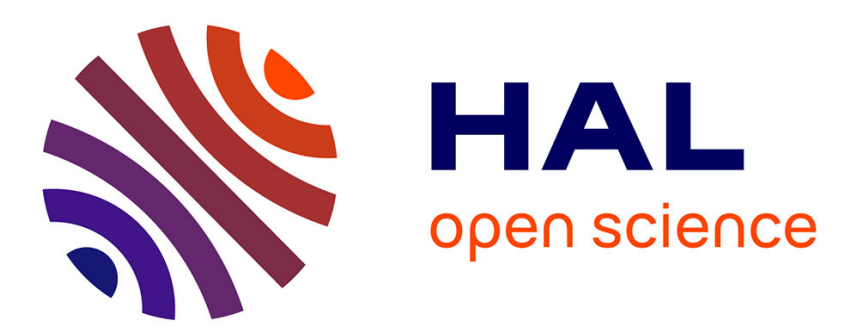

\title{
Cell-centered discontinuous Galerkin discretizations for two-dimensional scalar conservation laws on unstructured grids and for the one-dimensional Lagrangian hydrodynamics
}

\author{
François Vilar, P.H. Maire, Remi Abgrall
}

\section{To cite this version:}

François Vilar, P.H. Maire, Remi Abgrall. Cell-centered discontinuous Galerkin discretizations for twodimensional scalar conservation laws on unstructured grids and for the one-dimensional Lagrangian hydrodynamics. Computers and Fluids, 2010, pp.Article in Press. 10.1016/j.compfluid.2010.07.018 . inria-00538165

\section{HAL Id: inria-00538165 \\ https://hal.inria.fr/inria-00538165}

Submitted on 22 Nov 2010

HAL is a multi-disciplinary open access archive for the deposit and dissemination of scientific research documents, whether they are published or not. The documents may come from teaching and research institutions in France or abroad, or from public or private research centers.
L'archive ouverte pluridisciplinaire HAL, est destinée au dépôt et à la diffusion de documents scientifiques de niveau recherche, publiés ou non, émanant des établissements d'enseignement et de recherche français ou étrangers, des laboratoires publics ou privés. 


\title{
Cell-centered discontinuous Galerkin discretizations for two-dimensional scalar conservation laws on unstructured grids and for one-dimensional Lagrangian hydrodynamics
}

\author{
François Vilar $^{\mathrm{a}}$, Pierre-Henri Maire ${ }^{\mathrm{a}, *}$, Rémi Abgrall ${ }^{\mathrm{b}}$ \\ ${ }^{a}$ CEA CESTA, BP 2, 33114 Le Barp, France \\ ${ }^{b}$ INRIA and University of Bordeaux, Team Bacchus, Institut de Mathématiques de \\ Bordeaux, 351 Cours de la Libération, 33405 Talence Cedex, France
}

\begin{abstract}
We present cell-centered discontinuous Galerkin discretizations for two-dimensional scalar conservation laws on unstructured grids and also for the one-dimensional Lagrangian hydrodynamics up to third-order. We also demonstrate that a proper choice of the numerical fluxes allows to enforce stability properties of our discretizations.
\end{abstract}

Keywords: DG schemes, Lagrangian hydrodynamics, hyperbolic conservation laws, slope limiting

\section{Introduction}

The discontinuous Galerkin (DG) methods are locally conservative, stable and high-order accurate methods which represent one of the most promising current trends in computational fluid dynamics [3, 4]. They can be viewed as a natural high-order extension of the classical finite volume methods. This extension is constructed by means of a local variational formulation in each cell, which makes use of a piecewise polynomial approximation of the unknowns. In the present work, we describe cell-centered DG methods up to

\footnotetext{
${ }^{*}$ Corresponding author

Email addresses: vilar@celia.u-bordeaux1.fr (François Vilar), maire@celia.u-bordeaux1.fr (Pierre-Henri Maire), remi.abgrall@math.u-bordeaux1.fr (Rémi Abgrall)
} 
third-order not only for two-dimensional scalar conservation laws on general unstructured grids but also for the one-dimensional system of gas dynamics equations written in the Lagrangian form. In this particular formalism, a computational cell moves with the fluid velocity, its mass being constant, thus contact discontinuity are captured very sharply. The main feature of our DG method consists in using a local Taylor basis to express the approximate solution in terms of cell averages and derivatives at cell centroids [7]. The explicit Runge-Kutta method that preserves the total variation diminishing property of a one-dimensional space discretization is employed to perform the time discretization up to third order [3]. The monotonicity is enforced by limiting the coefficients in the Taylor expansion in a hierarchical manner using the vertex based slope limiter developed in $[7,11]$. We will first illustrate the robustness and the accuracy of this scheme by testing it against analytical solutions for simple conservation laws problems. Then, we will explore its performance in the Lagrangian framework by applying it in one-dimension. We note that in the case of systems, the limitation procedure is applied using the characteristic variables. Extending the methodology described in [2, 10], we derive numerical fluxes which enforce the stability in $L^{2}$ norm for the case of scalar conservation laws and provide an entropy inequality in the case of gas dynamics equations.

\section{Scalar conservation laws}

We develop our cell-centered DG method in the case of one and twodimensional scalar conservation laws.

\subsection{One-dimensional case}

Let $u=u(x, t)$, for $x \in \mathbb{R}$ and $t \geq 0$, be the solution of the following one-dimensional scalar conservation law

$$
\frac{\partial u}{\partial t}+\frac{\partial f(u)}{\partial x}=0, u(x, 0)=u^{0}(x)
$$

where $u^{0}$ is the initial data and $f(u)$ is the flux function.

\subsubsection{Discretization}

The DG discretization can be viewed as an extension of the finite volume method wherein a piecewise polynomial approximation of the unknown is used. Let us introduce $C_{i}=\left[x_{i-\frac{1}{2}}, x_{i+\frac{1}{2}}\right]$ a generic cell of size $\Delta x_{i}$ and $\mathbb{P}^{K}\left(C_{i}\right)$ 
the set of polynomials of degree up to $K$. We can express the numerical solution as

$$
u_{h}^{i}(x, t)=\sum_{k=0}^{K} u_{k}^{i}(t) e_{k}^{i}(x),
$$

where $\left\{e_{k}\right\}_{k=0 \ldots K}$ is a basis of $\mathbb{P}^{K}\left(C_{i}\right)$. The coefficients $u_{k}^{i}(t)$ are determined by writing the local variational formulation for $k=0 \ldots K$

$$
\sum_{l=0}^{K} \frac{d u_{l}^{i}}{d t} \int_{C_{i}}\left(e_{l}^{i}, e_{k}^{i}\right) \mathrm{d} x+\left[\overline{f(u)} e_{k}^{i}\right]_{x_{i-\frac{1}{2}}}^{x_{i+\frac{1}{2}}}-\int_{C_{i}} f\left(u_{h}^{i}\right) \frac{d e_{k}^{i}}{d x} \mathrm{~d} x=0 .
$$

Here, $\left[\overline{f(u)} e_{k}^{i}\right]_{x_{i-\frac{1}{2}}}^{x_{i+\frac{1}{2}}}=\bar{f}_{i+\frac{1}{2}} e_{k}^{i}\left(x_{i+\frac{1}{2}}^{-}\right)-\bar{f}_{i-\frac{1}{2}} e_{k}^{i}\left(x_{i-\frac{1}{2}}^{+}\right)$where $\bar{f}_{i+\frac{1}{2}}$ is again the numerical flux, which is a single valued function defined at the cell interfaces and in general depends on the numerical values of the numerical solution from both sides of the interface. Finally, substituting the projection of $f\left(u_{h}^{i}\right)$ onto the approximation space into (2) leads to

$$
\mathbf{M}^{i} \frac{d}{d t} \boldsymbol{U}^{i}+\left[\overline{f(u)}(x) \boldsymbol{B}^{i}(x)\right]_{x_{i-\frac{1}{2}}}^{x_{i+\frac{1}{2}}}-\mathrm{D}^{i} \boldsymbol{F}^{i}=0,
$$

where $\mathrm{M}_{k l}^{i}=\int_{C_{i}}\left(e_{k}^{i}, e_{l}^{i}\right) \mathrm{d} x$ is the mass matrix, $\boldsymbol{U}^{i}=\left(u_{0}^{i}, \ldots, u_{l}^{i}, \ldots, u_{K}^{i}\right)^{T}$ is the unknown vector, $\boldsymbol{B}^{i}(x)=\left(e_{0}^{i}(x), \ldots, e_{l}^{i}(x), \ldots, e_{K}^{i}(x)\right)^{T}, \boldsymbol{F}^{i}=\left(f_{0}^{i}, \ldots, f_{l}^{i}, \ldots, f_{K}^{i}\right)^{T}$ and $\mathrm{D}_{k l}^{i}=\int_{C_{i}}\left(\partial_{x} e_{k}^{i}, e_{l}^{i}\right) \mathrm{d} x$. To achieve the discretization, we define the local Taylor basis $\left\{e_{k}\right\}_{k=0 \ldots K}$ by setting

$$
e_{k}^{i}=\frac{1}{k !}\left[\left(\frac{x-x_{i}}{\Delta x_{i}}\right)^{k}-\left\langle\left(\frac{x-x_{i}}{\Delta x_{i}}\right)^{k}\right\rangle\right]
$$

where $\langle\phi\rangle$ denotes the mean value of $\phi$ over the cell $C_{i}$ and $x_{i}$ is the midpoint of $C_{i}$. We point out that the projection of a smooth function over this Taylor basis is strongly related to its Taylor expansion at the cell center $x_{i}$. More precisely, for $K=2$, the approximate solution $u_{h}^{i}$ reads

$$
u_{h}^{i}(x, t)=u_{0}^{i}(t)+u_{1}^{i}(t)\left(\frac{x-x_{i}}{\Delta x_{i}}\right)+u_{2}^{i}(t) \frac{1}{2}\left[\left(\frac{x-x_{i}}{\Delta x_{i}}\right)^{2}-\frac{1}{12}\right],
$$

where $u_{0}^{i}=\langle u\rangle$ and $u_{k}^{i}=\left\langle\frac{\partial^{k} u}{\partial x^{k}}\right\rangle \Delta x_{i}^{k}$. The time discretization of (3) utilizes the classical third-order TVD Runge-Kutta scheme [10]. 


\subsubsection{Numerical flux and $L^{2}$ stability}

Following $[2,10]$, we provide a numerical flux which ensures the stability of our discretization in the $L^{2}$ norm. To this end, let us consider the local variational formulation written using $u_{h}^{i}$ as a test function

$$
\frac{1}{2} \frac{d}{d t} \int_{C_{i}}\left(u_{h}^{i}\right)^{2} \mathrm{~d} x+\left[\overline{f\left(u_{h}^{i}\right)} u_{h}^{i}\right]_{x_{i-\frac{1}{2}}}^{x_{i+\frac{1}{2}}}-\left[F\left(u_{h}^{i}\right)\right]_{x_{i-\frac{1}{2}}}^{x_{i+\frac{1}{2}}}=0
$$

Here, we make use of the function $F$ which denotes a primitive of the flux function defined as $F(u)=\int_{0}^{u} f(s) \mathrm{d} s$. Let us set $R_{i}=\left[\overline{f\left(u_{h}^{i}\right)} u_{h}^{i}\right]_{x_{i-\frac{1}{2}}}^{x_{i+\frac{1}{2}}}-$ $\left[F\left(u_{h}^{i}\right)\right]_{x_{i-\frac{1}{2}}}^{x_{i+\frac{1}{2}}}$. For periodic boundary conditions, the sum of (5) over all the cells writes

$$
\frac{1}{2} \frac{d}{d t} \sum_{i, \text { cells }} \int_{C_{i}}\left(u_{h}^{i}\right)^{2} \mathrm{~d} x+\sum_{i, c e l l s} R_{i}=0 .
$$

At this point, we claim that the stability in $L^{2}$ norm for our semi-discrete scheme amounts to impose

$$
\sum_{i, \text { cells }} R_{i} \geq 0
$$

Next, we determine the form of the numerical flux so that (7) is enforced. By interchanging the sum from cells to nodes, (7) re-writes

$$
\sum_{i, \text { cells }} R_{i}=\sum_{i, \text { nodes }}\left(u_{L}-u_{R}\right)\left(\bar{f}_{i+\frac{1}{2}}-\frac{1}{u_{R}-u_{L}} \int_{u_{L}}^{u_{R}} f(u) \mathrm{d} u\right),
$$

where $u_{L}$ and $u_{R}$ denote the left and right states on both sides of the interface, i.e. $u_{L}=u_{h}^{i}\left(x_{i+\frac{1}{2}}^{-}\right)$and $u_{R}=u_{h}^{i+1}\left(x_{i+\frac{1}{2}}^{+}\right)$. Finally, the stability of the semidiscrete scheme in $L^{2}$ norm is ensured provided that the numerical flux is written

$$
\bar{f}_{i+\frac{1}{2}}=\frac{1}{u_{R}-u_{L}} \int_{u_{L}}^{u_{R}} f(u) \mathrm{d} u-C_{i+\frac{1}{2}}\left(u_{R}-u_{L}\right)
$$

where $C_{i+\frac{1}{2}}$ is a positive scalar which has the physical dimension of a velocity. In the linear case, $f(u)=a u$, where $a$ is the constant advection velocity, we get $\bar{f}_{i+\frac{1}{2}}=\frac{a}{2}\left(u_{L}+u_{R}\right)-C_{i+\frac{1}{2}}\left(u_{R}-u_{L}\right)$. We recognize two different parts in this flux, the centered one $\frac{a}{2}\left(u_{L}+u_{R}\right)$, and the viscous one $C_{i+\frac{1}{2}}\left(u_{R}-u_{L}\right)$ which brings dissipation and consequently stability. We also note that for $C_{i+\frac{1}{2}}=\frac{|a|}{2}$ we recover the well known upwind scheme, whereas for $C_{i+\frac{1}{2}}=\frac{\Delta x_{i}}{2 \Delta t}$ 
we get the Lax-Friedrichs scheme. In non-linear case, we can use a quadrature formula to evaluate $\int_{u_{L}}^{u_{R}} f(u) \mathrm{d} u$. If we choose the trapezoidal rule and take $C_{i+\frac{1}{2}}=\frac{1}{2} \max \left(\left|f^{\prime}\left(u_{L}\right)\right|,\left|f^{\prime}\left(u_{R}\right)\right|\right)$, we recover the local Lax-Friedrichs scheme

$$
\bar{f}_{i+\frac{1}{2}}=\frac{f\left(u_{L}\right)+f\left(u_{R}\right)}{2}-\max \left(\left|f^{\prime}\left(u_{L}\right)\right|,\left|f^{\prime}\left(u_{R}\right)\right|\right)\left(\frac{u_{R}-u_{L}}{2}\right) .
$$

We notice that if $f(u)=a u$, where $a$ is a constant velocity, the local LaxFriedrichs flux reduces to the classical upwind flux. We also remark that the proof of the $L^{2}$ stability presented above has been already derived in $[5,6]$.

\subsubsection{Limitation}

Following Kuzmin [7], we define a hierarchical limiting procedure by multiplying all derivatives of order $k$ by a factor $\alpha_{k}$. Thus the limited counterpart of the approximate solution (4) writes

$$
u_{h}^{i}(x)=u_{0}^{i}+\alpha_{1}^{i} u_{1}^{i}\left(\frac{x-x_{i}}{\Delta x_{i}}\right)+\alpha_{2}^{i} u_{2}^{i} \frac{1}{2}\left[\left(\frac{x-x_{i}}{\Delta x_{i}}\right)^{2}-\frac{1}{12}\right] .
$$

The coefficients $\alpha_{1}^{i}$ and $\alpha_{2}^{i}$ are determined using the vertex-based limiter defined in [7]. That is, we want the extrapolated value at a generic node to be bounded by the minimum and maximum averaged values taken over the cells surrounding this node. We apply this procedure to the linear reconstructions

$$
\Delta x_{i} \frac{\partial u_{h}^{i}}{\partial x}=u_{1}^{i}+\alpha_{2}^{i} u_{2}^{i}\left(\frac{x-x_{i}}{\Delta x_{i}}\right), \quad\left(u_{h}^{i}\right)^{1}=u_{0}^{i}+\alpha_{1}^{i} u_{1}^{i}\left(\frac{x-x_{i}}{\Delta x_{i}}\right) .
$$

To preserve smooth extrema, we set $\alpha_{1}^{i}=\max \left(\alpha_{1}^{i}, \alpha_{2}^{i}\right)$. We note that this limiter is a moment based limiter as the ones described in $[1,11]$.

\subsubsection{Numerical results}

We have checked the order of convergence of our DG scheme for the nonlinear Burgers equation $\left(f(u)=u^{2} / 2\right)$, using the smooth initial condition $u_{0}(x)=\sin (2 \pi x)$ over the domain $[0,1]$ with periodic boundary conditions. The analytical solution is computed using the method of characteristics prior to shock formation at time $t=\frac{1}{2 \pi}$. The results displayed in Table 1 illustrate the accuracy of our discretization. To demonstrate the performance of the hierarchical slope limiter, we have run the test case described in [11], which consists in advecting a combination of smooth and discontinuous profiles 


\begin{tabular}{|c||c|c|c|c|}
\hline & & $L_{1}$ & $L_{2}$ & $L_{\infty}$ \\
\hline \hline \multirow{4}{*}{ Burgers } & first-order & 0.86 & 0.68 & 0.23 \\
\cline { 2 - 5 } & second-order & 2.00 & 1.99 & 1.91 \\
\cline { 2 - 5 } & second-order lim & 2.12 & 1.99 & 1.57 \\
\cline { 2 - 5 } & third-order & 2.88 & 2.91 & 2.65 \\
\cline { 2 - 5 } & third-order lim & 2.87 & 2.89 & 2.62 \\
\hline
\end{tabular}

Table 1: Convergence rate for smooth solution of Burgers equation.

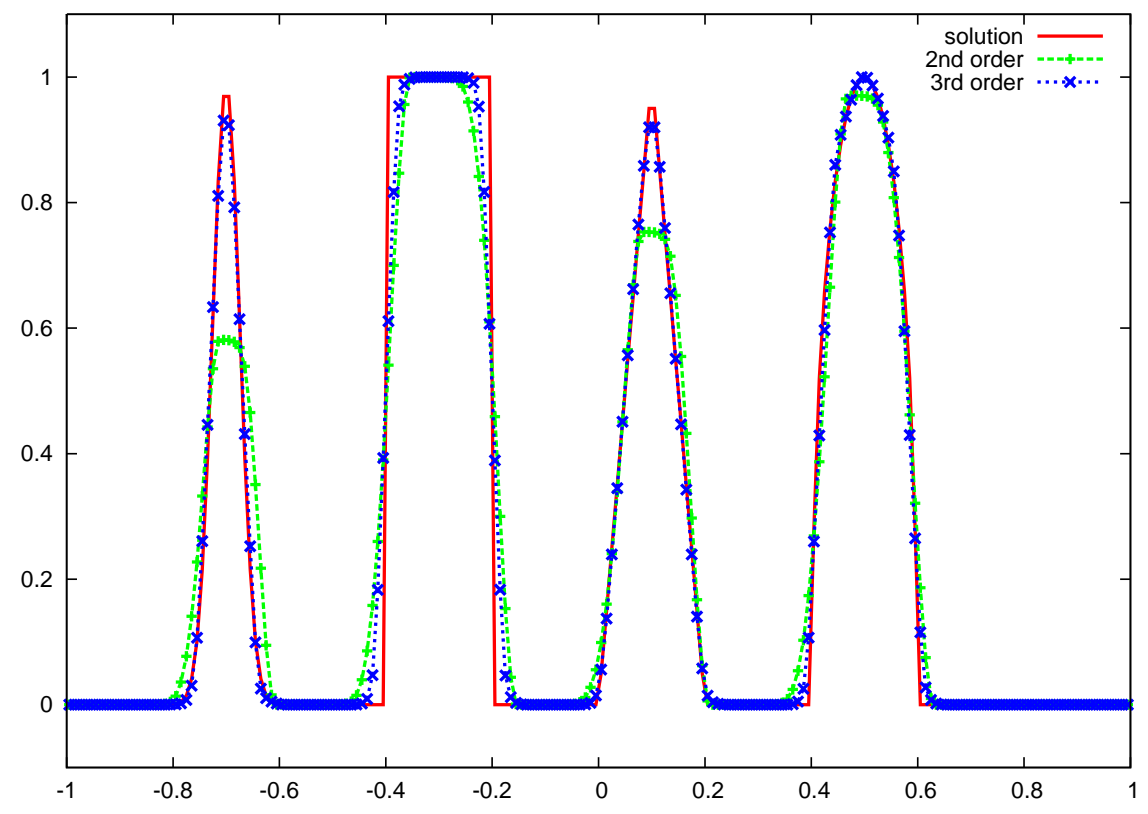

Figure 1: Linear advection of a combination of smooth and discontinuous profiles. Comparison between the second-order and the third-order scheme.

using periodic boundary conditions over the domain $[-1,1]$. The results obtained for the second and third order schemes at time $T=8$ are displayed in Figure 1. They show that the smooth extrema are perfectly preserved for the third-order scheme. 


\subsection{Two-dimensional linear case}

Let us now describe our cell-centered DG method for two-dimensional scalar conservation laws on unstructured grids. Let $u=u(\boldsymbol{x}, t)$ be the solution of the following two-dimensional scalar conservation law, for $\boldsymbol{x} \in \mathbb{R}^{2}$ and $t \geq 0$

$$
\frac{\partial u}{\partial t}+\nabla \cdot \boldsymbol{f}(u)=0, u(\boldsymbol{x}, 0)=u^{0}(\boldsymbol{x}),
$$

where $u^{0}$ is the initial data and $\boldsymbol{f}(u)=\left(f_{1}(u), f_{2}(u)\right)^{T}$ with $f_{1}(u)$ and $f_{2}(u)$ are the two directional fluxes.

\subsubsection{Discretization}

Using the same approach than for the one-dimensional case, we obtain a similar compact equation

$$
\mathrm{M}^{i} \frac{d}{d t} \boldsymbol{U}^{i}+\int_{\partial C_{i}} \overline{\boldsymbol{f}(u)} \cdot \boldsymbol{n} \boldsymbol{B}^{i} \mathrm{~d} \Gamma-\mathrm{D}_{1}{ }^{i} \boldsymbol{F}_{\mathbf{1}}{ }^{i}-\mathrm{D}_{2}{ }^{i} \boldsymbol{F}_{\mathbf{2}}{ }^{i}=0,
$$

where $\boldsymbol{n}$ denotes the outward unit normal to the cell interface $\Gamma . \mathrm{M}_{k l}^{i}=$ $\int_{C_{i}}\left(e_{k}^{i}, e_{l}^{i}\right) \mathrm{d} C$ is the mass matrix, $\boldsymbol{U}^{i}=\left(u_{0}^{i}, \ldots, u_{l}^{i}, \ldots, u_{K}^{i}\right)^{T}$ our unknown vector, $\mathbf{D}_{\mathrm{j} k l}^{i}=\int_{C_{i}}\left(\partial_{j} e_{k}^{i}, e_{l}^{i}\right) \mathrm{d} C, \boldsymbol{B}^{i}(\boldsymbol{x})=\left(e_{0}^{i}(\boldsymbol{x}), \ldots, e_{l}^{i}(\boldsymbol{x}), \ldots, e_{K}^{i}(\boldsymbol{x})\right)^{T}$ and $\boldsymbol{F}_{j}{ }^{i}=\left(f_{j, 0}^{i}, \ldots, f_{j, l}^{i}, \ldots, f_{j, K}^{i}\right)^{T}$. The unknowns to be solved in this formulation are the cell-averaged variables and their derivatives at the center of the cells, regardless of elements shape. For the third-order scheme, the dimension of the polynomial space is six and the six basis functions are $e_{0}^{i}=1, e_{1}^{i}=\frac{x-x_{i}}{\Delta x_{i}}$, $e_{2}^{i}=\frac{y-y_{i}}{\Delta y_{i}}, e_{3}^{i}=\frac{1}{2}\left[\left(\frac{x-x_{i}}{\Delta x_{i}}\right)^{2}-\left\langle\left(\frac{x-x_{i}}{\Delta x_{i}}\right)^{2}\right\rangle\right], e_{4}^{i}=\frac{\left(x-x_{i}\right)\left(y-y_{i}\right)}{\Delta x_{i} \Delta y_{i}}-\left\langle\frac{\left(x-x_{i}\right)\left(y-y_{i}\right)}{\Delta x_{i} \Delta y_{i}}\right\rangle$, $e_{5}^{i}=\frac{1}{2}\left[\left(\frac{y-y_{i}}{\Delta y_{i}}\right)^{2}-\left\langle\left(\frac{y-y_{i}}{\Delta y_{i}}\right)^{2}\right\rangle\right]$, where $\Delta x_{i}=0.5\left(x_{\max }-x_{\min }\right)$ and $\Delta y_{i}=$ $0.5\left(y_{\max }-y_{\min }\right)$ and $x_{\max }, y_{\max }, x_{\min }, y_{\min }$ are the maximum and the minimum x and y-coordinates in the cell $C_{i}$.

\subsubsection{Numerical flux and $L^{2}$ stability}

Following $[2,10]$ we design a numerical flux which ensures $L^{2}$ stability. To this end, let us consider the variational formulation

$$
\frac{1}{2} \frac{d}{d t} \int_{C_{i}} u_{h}^{2} \mathrm{~d} C+\int_{\partial C_{i}}\left(\overline{\boldsymbol{f}(u)} u_{h}-\boldsymbol{F}\left(u_{h}\right)\right) \cdot \boldsymbol{n} \mathrm{d} \Gamma=0,
$$

where we have set $\boldsymbol{F}(u)=\left(\int_{0}^{u} f_{1}(s) \mathrm{d} s, \int_{0}^{u} f_{2}(s) \mathrm{d} s\right)^{T}$. Introducing $R_{i}=$ $\int_{\partial C_{i}}\left(\overline{\boldsymbol{f}(u)} u_{h}-\boldsymbol{F}\left(u_{h}\right)\right) \cdot \boldsymbol{n} \mathrm{d} \Gamma$ and summing over all the cells, we finally obtain 
the following positivity condition on $R_{i}$ in order to ensure the $L^{2}$ stability of our semi-discrete scheme

$$
\sum_{i, \text { cells }} R_{i}=\sum_{i, \text { cells }} \sum_{f_{e} \in \text { face }\left(C_{i}\right)} \int_{f_{e}}\left(\overline{\boldsymbol{f}(u)}^{f_{e}} u_{h}-\boldsymbol{F}\left(u_{h}\right)\right) \cdot \boldsymbol{n}_{f_{e}} \mathrm{~d} \Gamma \geq 0 .
$$

To design a numerical flux which enforces (13), we interchange the sums from cells to faces to get

$$
\sum_{i} R_{i}=\sum_{f_{e}} \int_{f_{e}}\left[\overline{\boldsymbol{f}(u}^{f_{e}}\left(u_{L}-u_{R}\right)-\left(\boldsymbol{F}\left(u_{L}\right)-\boldsymbol{F}\left(u_{R}\right)\right)\right] \cdot \boldsymbol{n}_{f_{e}} \mathrm{~d} \Gamma .
$$

Here, $u_{L}$ and $u_{R}$ denote the extrapolated values of the variable $u$ on both sides of the interface $f_{e}$. Namely, if $\boldsymbol{x}_{f_{e}}$ denotes a point located on $f_{e}$, then $u_{L}=\lim _{\lambda \rightarrow 0^{+}} u_{h}\left(\boldsymbol{x}_{f_{e}}-\lambda \boldsymbol{n}_{f_{e}}\right)$ and $u_{R}=\lim _{\lambda \rightarrow 0^{+}} u_{h}\left(\boldsymbol{x}_{f_{e}}+\lambda \boldsymbol{n}_{f_{e}}\right)$. Finally, the stability of the semi-discrete scheme in $L^{2}$ norm is ensured provided that the numerical flux is written

$$
\overline{\boldsymbol{f}(u)})^{f_{e}}=\frac{1}{u_{R}-u_{L}} \int_{u_{L}}^{u_{R}} \boldsymbol{f}(s) \mathrm{d} s-\left(u_{R}-u_{L}\right) \mathrm{M}_{f_{e}} \boldsymbol{n}_{f_{e}},
$$

where $\mathrm{M}_{f_{e}}$ is a positive definite matrix which has the physical dimension of a velocity. For linear case, $\boldsymbol{f}(u)=\boldsymbol{A} u$, where $\boldsymbol{A}$ is the constant advection velocity, we get $\overline{\boldsymbol{f}(u)}{ }^{f_{e}}=\frac{u_{L}+u_{R}}{2} \boldsymbol{A}-\left(u_{R}-u_{L}\right) \mathrm{M}_{f_{e}} \boldsymbol{n}_{f_{e}}$. By setting $\mathrm{M}_{f_{e}}=$ $\mathrm{M}_{f_{e}}^{1}=\frac{1}{2}\left|\boldsymbol{A} \cdot \boldsymbol{n}_{f_{e}}\right|$ Id, we recover the classical upwind scheme. Note that by setting $\mathrm{M}_{f_{e}}=\mathrm{M}_{f_{e}}^{2}=\frac{1}{2}\left|\boldsymbol{A} \cdot \boldsymbol{n}_{f_{e}}\right| \frac{\boldsymbol{A} \otimes \boldsymbol{A}}{\|\boldsymbol{A}\|^{2}}$, we define a less dissipative scheme since

$$
\frac{\left(\mathrm{M}_{f_{e}}^{2} \boldsymbol{n}_{f_{e}}, \boldsymbol{n}_{f_{e}}\right)}{\left(\mathrm{M}_{f_{e}}^{1} \boldsymbol{n}_{f_{e}}, \boldsymbol{n}_{f_{e}}\right)}=(\cos \theta)^{2} \leq 1
$$

where $\theta$ is the angle between $\boldsymbol{A}$ and $\boldsymbol{n}_{f_{e}}$. In the general non-linear case, as in the one-dimensional study, a quadrature formula can be used. Taking the same trapezoidal rule and $\mathrm{M}_{f_{e}}=\frac{1}{2} \max \left(\left|\boldsymbol{A}\left(u_{L}\right) \cdot \boldsymbol{n}_{f_{e}}\right|,\left|\boldsymbol{A}\left(u_{R}\right) \cdot \boldsymbol{n}_{f_{e}}\right|\right)$, we recover the local Lax-Friedrichs scheme

$\overline{\boldsymbol{f}(u)}^{f_{e}} \cdot \boldsymbol{n}_{f_{e}}=\frac{1}{2}\left(\boldsymbol{f}\left(u_{L}\right)+\boldsymbol{f}\left(u_{R}\right)\right) \cdot \boldsymbol{n}_{f_{e}}-\max \left(\left|\boldsymbol{A}\left(u_{L}\right) \cdot \boldsymbol{n}_{f_{e}}\right|,\left|\boldsymbol{A}\left(u_{R}\right) \cdot \boldsymbol{n}_{f_{e}}\right|\right)\left(\frac{u_{R}-u_{L}}{2}\right)$,

where $\boldsymbol{A}(u) \equiv \frac{d}{d u} \boldsymbol{f}(u)=\left(f_{1}^{\prime}(u), f_{2}^{\prime}(u)\right)^{T}$. 


\begin{tabular}{|c||c|c|c|}
\hline \multicolumn{1}{|c||}{} & & $L_{1}$ & $L_{2}$ \\
\hline \hline \multirow{4}{*}{ Linear advection } & first-order & 1.02 & 1.02 \\
\cline { 2 - 4 } & second-order & 1.99 & 1.98 \\
\cline { 2 - 4 } & second-order lim & 2.15 & 2.15 \\
\cline { 2 - 4 } & third-order & 2.98 & 2.98 \\
\cline { 2 - 4 } & third-order $\lim$ & 3.45 & 3.22 \\
\hline
\end{tabular}

Table 2: Convergence rate for linear advection with and without slope limitation for the smooth initial condition $u_{0}(\boldsymbol{x})=\sin (2 \pi x) \sin (2 \pi y)$ on a sequence of Cartesian grids.

\subsubsection{Numerical results}

Linear advection. First, we assess the accuracy of our DG scheme by computing the order of convergence for a smooth initial condition using a velocity field corresponding to a rigid rotation defined by $\boldsymbol{A}=(0.5-y, x-0.5)^{T}$. The results displayed in Table 2 demonstrate the expected order of convergence is reached even with limitation. Following [7], we compute the solid body rotation test case using the same velocity field. The numerical solution, plotted in Figure 2 exhibits quite similar results than those obtained in [7].

KPP rotating wave problem. Here, we consider the non-linear KPP problem taken from [8]. For this particular problem, the fluxes are non-convex and defined by $f_{1}(u)=\sin (u), f_{2}(u)=\cos (u)$. The computational domain $[-2,2] \times[-2.5,1.5]$ is paved using polygonal cells which result from a Voronoi tessellation. Initial condition is defined by

$$
u_{0}(\boldsymbol{x})= \begin{cases}\frac{7 \pi}{2} & \text { if } \sqrt{x^{2}+y^{2}} \leq 1 \\ \frac{\pi}{4} & \text { if } \sqrt{x^{2}+y^{2}}>1\end{cases}
$$

The numerical result obtained using a general unstructured grid made of 2500 polygonal cells, with the third-order DG scheme, is very similar to the result obtained in [8]. It exhibits the correct composite wave structure. 


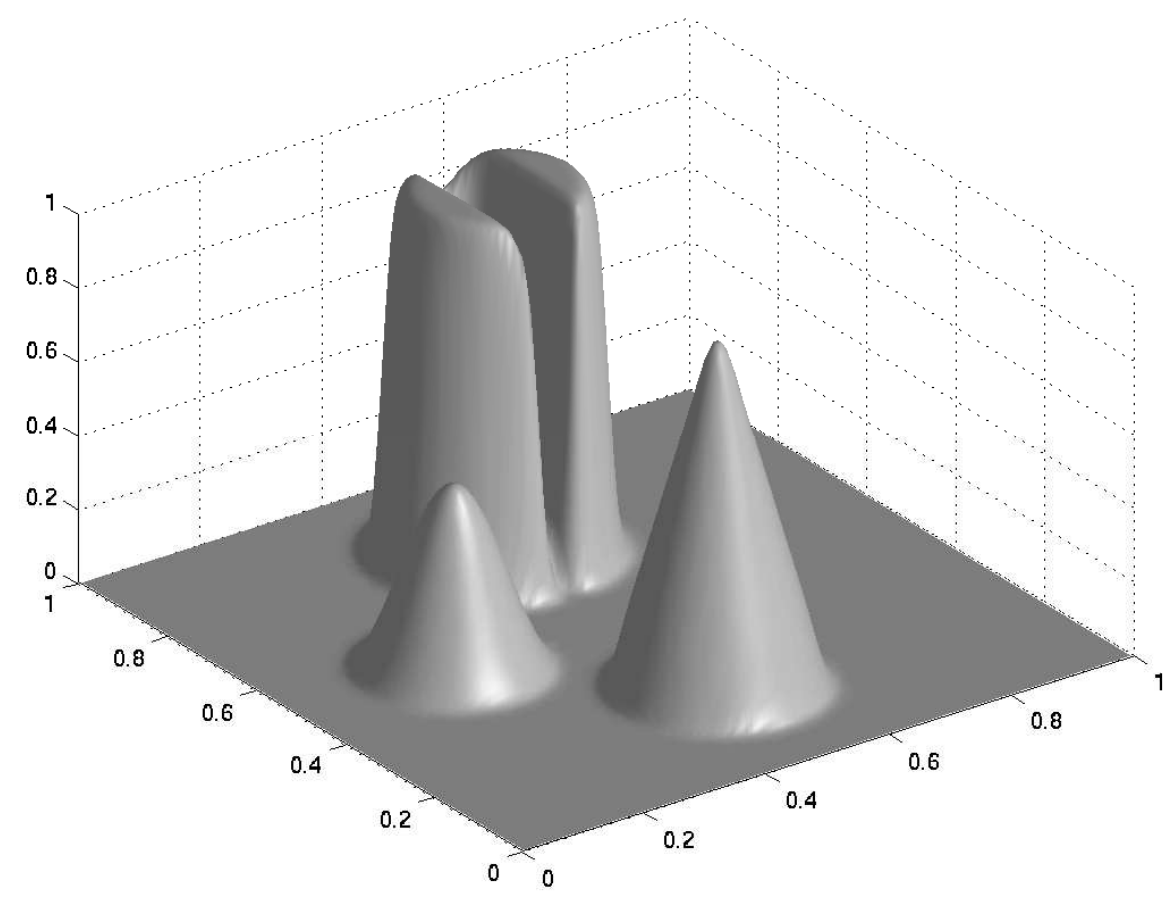

Figure 2: Numerical solutions for the solid body rotation test case [7], with third-order GD and limitation for a $128 \times 128$ Cartesian grid. The $L^{1}$ and $L^{2}$ norms of the global truncation error are $E_{1}=1.49 e-2, E_{2}=6.61 e-2$. 


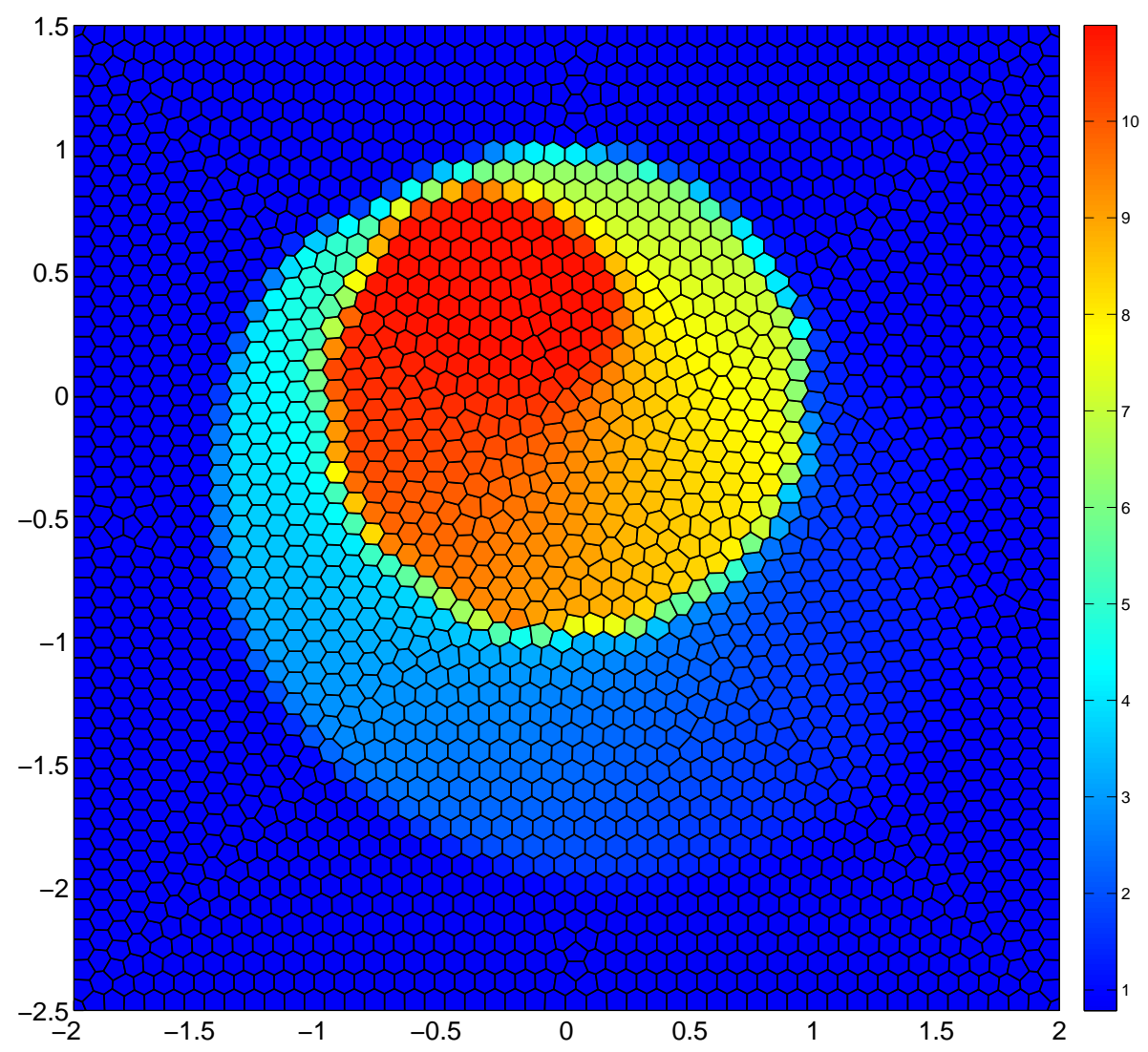

Figure 3: Numerical solution for the KPP problem at time $t=1$, using third-order limited DG on a polygonal grid made of 2500 cells. 


\section{One-dimensional Lagrange hydrodynamics}

In this section, we solve the one-dimensional gas dynamics equations written in Lagrangian formalism

$$
\begin{aligned}
& \rho^{0} \frac{d}{d t}\left(\frac{1}{\rho}\right)-\frac{\partial u}{\partial x}=0, \\
& \rho^{0} \frac{d u}{d t}+\frac{\partial p}{\partial x}=0, \\
& \rho^{0} \frac{d E}{d t}+\frac{\partial(p u)}{\partial x}=0,
\end{aligned}
$$

where $\rho$ is the density of the fluid, $\rho^{0}>0$ its initial density, $u$ its velocity and $E$ its total energy. Here $x$ denotes the Lagrangian coordinate. The thermodynamic closure of this system is obtained through the use of an equation of state, which writes $p=p(\rho, \varepsilon)$ where $\varepsilon$ is the specific internal energy, $\varepsilon=E-\frac{u^{2}}{2}$. For numerical application, we use a gamma gas law, i.e. $p=\rho(\gamma-1) \varepsilon$ where $\gamma$ is the polytropic index of the gas.

\subsection{Flux and entropy inequality}

The aim of this section is to design numerical flux so that our semidiscrete DG scheme satisfies a global entropy inequality. If $\phi$ denotes an exact solution of the previous system, we denote by $\phi_{h}$ its piecewise polynomial approximation. Namely, the restriction of $\phi_{h}$ over the cell $C_{i}=\left[x_{i-\frac{1}{2}}, x_{i+\frac{1}{2}}\right]$ is a polynomial. To construct a variational formulation of the previous system, we multiply respectively (16a), (16b) and (16c) by the test functions $p_{h}$, $u_{h}$ and $1_{h}$, integrate over $C_{i}$ and replace the exact solution $\left(\frac{1}{\rho}, u, E\right)$ by its approximation $\left[\left(\frac{1}{\rho}\right)_{h}, u_{h}, E_{h}\right]$ :

$$
\begin{aligned}
& \int_{C_{i}} \rho_{h}^{0} p_{h} \frac{d}{d t}\left(\frac{1}{\rho}\right)_{h} \mathrm{~d} x=\left[p_{h} \bar{u}\right]_{x_{i-\frac{1}{2}}}^{x_{i+\frac{1}{2}}}-\int_{C_{i}} u_{h} \frac{\partial p_{h}}{\partial x} \mathrm{~d} x, \\
& \int_{C_{i}} \rho_{h}^{0} u_{h} \frac{d}{d t} u_{h} \mathrm{~d} x=-\left[\bar{p} u_{h}\right]_{x_{i-\frac{1}{2}}}^{x_{i+\frac{1}{2}}}+\int_{C_{i}} p_{h} \frac{\partial u_{h}}{\partial x} \mathrm{~d} x, \\
& \int_{C_{i}} \rho_{h}^{0} \frac{d}{d t} E_{h} \mathrm{~d} x=-[\overline{p u}]_{x_{i-\frac{1}{2}}}^{x_{i+\frac{1}{2}}} .
\end{aligned}
$$

Here, $\bar{u}, \bar{p}$ and $\overline{p u}$ are the numerical fluxes that we look for. We note that the polynomial approximation of the pressure, $p_{h}$, is obtained through the use 
of an orthogonal projection onto the polynomial basis using the pointwise defined equation of state. The combination (17c)-(17b)+(17a) leads to

$$
\begin{aligned}
\int_{C_{i}} \rho_{h}^{0}\left[\frac{d}{d t}\left(E_{h}-\frac{1}{2} u_{h}^{2}\right)+p_{h} \frac{d}{d t}\left(\frac{1}{\rho}\right)_{h}\right] \mathrm{d} x & =\left[p_{h} \bar{u}+\bar{p} u_{h}-\overline{p u}\right]_{x_{i-\frac{1}{2}}}^{x_{i+\frac{1}{2}}}-\int_{C_{i}}\left(p_{h} \frac{\partial u_{h}}{\partial x}+u_{h} \frac{\partial p_{h}}{\partial x}\right) \mathrm{d} x \\
& =\left[p_{h} \bar{u}+\bar{p} u_{h}-\overline{p u}-p_{h} u_{h}\right]_{x_{i-\frac{1}{2}}}^{x_{i+\frac{1}{2}}}
\end{aligned}
$$

Knowing that specific internal energy writes as $\varepsilon=E-\frac{1}{2} u^{2}$, and specific entropy is expressed according to the Gibbs formula as $T d S=d \varepsilon+p d\left(\frac{1}{\rho}\right)$, where $T$ denotes the temperature, we deduce

$$
\int_{C} \rho_{h}^{0} T_{h} \frac{d S_{h}}{d t} \mathrm{~d} x=\sum_{i, c e l l s} R_{i}
$$

where $R_{i}=\left[p_{h} \bar{u}+\bar{p} u_{h}-\overline{p u}-p_{h} u_{h}\right]_{x_{i-\frac{1}{2}}}^{x_{i+\frac{1}{2}}}$. At this point, it remains to express the numerical fluxes in such a way that an entropic inequality is satisfied. To this end, we first make the following fundamental assumption

$$
\overline{p u}=\bar{p} \bar{u} .
$$

This assumption allows to factorize $R_{i}$ and to write it as $R_{i}=[(p-\bar{p})(\bar{u}-$ $u)]_{x_{i-\frac{1}{2}}}^{x_{i+\frac{1}{2}}}$. Thus, entropy production related to the semi-discrete scheme writes

$$
\int_{C} \rho_{h}^{0} T_{h} \frac{d S_{h}}{d t} \mathrm{~d} x=\sum_{i, c e l l s}\left[\left(p_{h}-\bar{p}\right)\left(\bar{u}-u_{h}\right)\right]_{x_{i-\frac{1}{2}}}^{x_{i+\frac{1}{2}}} .
$$

We want our DG formulation to satisfy the second law of thermodynamics, that is we want it to convert kinetic energy into internal energy through shock waves. This amounts to design numerical fluxes so that $\sum_{i} R_{i} \geq 0$. Interchanging the sum from cells to nodes and setting $\phi_{h}\left(x_{i+\frac{1}{2}}^{-}\right)=\phi_{L}$ and $\phi_{h}\left(x_{i+\frac{1}{2}}^{+}\right)=\phi_{R}$ yields

$$
\sum_{i, \text { cells }} R_{i}=\sum_{i, \text { nodes }}\left[\left(p_{L}-\bar{p}_{i+\frac{1}{2}}\right)\left(\bar{u}_{i+\frac{1}{2}}-u_{L}\right)-\left(p_{R}-\bar{p}_{i+\frac{1}{2}}\right)\left(\bar{u}_{i+\frac{1}{2}}-u_{R}\right)\right] .
$$

Here, we note that the previous equation has been obtained using periodic boundary conditions. We claim that a sufficient condition to satisfy $\sum_{i} R_{i} \geq$ 
0 consists in setting

$$
\begin{aligned}
& \bar{p}_{i+\frac{1}{2}}=p_{R}+Z_{i+\frac{1}{2}}^{R}\left(\bar{u}_{i+\frac{1}{2}}-u_{R}\right), \\
& \bar{p}_{i+\frac{1}{2}}=p_{L}+Z_{i+\frac{1}{2}}^{L}\left(u_{L}-\bar{u}_{i+\frac{1}{2}}\right),
\end{aligned}
$$

where $Z_{i+\frac{1}{2}}^{L / R}$ are positive scalars which have the physical dimension of a density times a velocity. The numerical fluxes at node $x_{i+\frac{1}{2}}$ are obtained by solving the previous linear system

$$
\begin{aligned}
& \bar{p}_{i+\frac{1}{2}}=\frac{Z_{i+\frac{1}{2}}^{L} p_{R}+Z_{i+\frac{1}{2}}^{R} p_{L}}{Z_{i+\frac{1}{2}}^{L}+Z_{i+\frac{1}{2}}^{R}}-\frac{Z_{i+\frac{1}{2}}^{L} Z_{i+\frac{1}{2}}^{R}}{Z_{i+\frac{1}{2}}^{L}+Z_{i+\frac{1}{2}}^{R}}\left(u_{R}-u_{L}\right), \\
& \bar{u}_{i+\frac{1}{2}}=\frac{Z_{i+\frac{1}{2}}^{L} u_{L}+Z_{i+\frac{1}{2}}^{R} u_{R}}{Z_{i+\frac{1}{2}}^{L}+Z_{i+\frac{1}{2}}^{R}}-\frac{1}{Z_{i+\frac{1}{2}}^{L}+Z_{i+\frac{1}{2}}^{R}}\left(p_{R}-p_{L}\right) .
\end{aligned}
$$

By taking $Z=\rho C$, where $C$ is the sound speed, we recover the classical acoustic Godunov solver.

\subsection{Limitation}

Concerning the slope limitation, before trying to apply it on the gas dynamics system, we firstly focus on the acoustic waves one. We noticed that if we perform the limitation on the physical variables, some oscillations remain. On the other hand, if we limit the Riemann invariants, our solution is perfectly monotone. We see in Figure 4 that the oscillations are quite strong at the shock front, without any limitation. But, unlike the acoustic problem, our system is not linear anymore. We cannot find the Riemann invariants with the same procedure than before. In the case of a regular flow, we get three quantities that are the differentials of the Riemann invariants

$$
d J_{ \pm}=d u \mp \rho C d\left(\frac{1}{\rho}\right), d J_{0}=d E-u d u+p d\left(\frac{1}{\rho}\right) .
$$

Linearizing these quantities, on each cells, around the mean values in the cell yields

$$
J_{ \pm}^{i}=u_{h}^{i} \mp \rho_{0}^{i} C_{0}^{i}\left(\frac{1}{\rho}\right)_{h}^{i}, J_{0}^{i}=E_{h}^{i}-u_{0}^{i} u_{h}^{i}+p_{0}^{i}\left(\frac{1}{\rho}\right)_{h}^{i},
$$

where $\phi_{h}^{i}$ is the polynomial approximation of $\phi$ on the cell $C_{i}$ and $\phi_{0}^{i}$ its mean value. This procedure is equivalent to linearize the equations, on each 


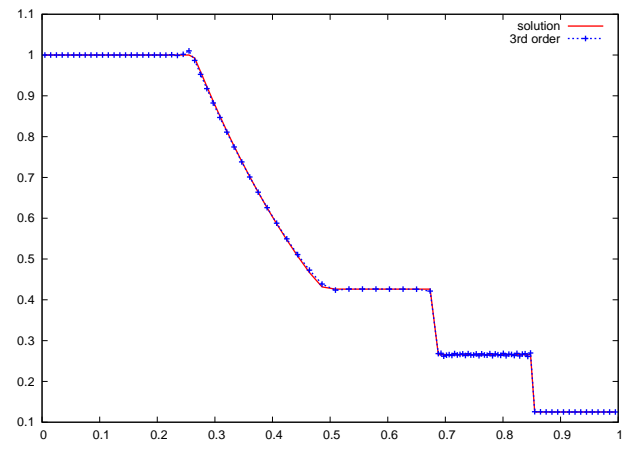

(a) Third-order DG for the Sod shock tube problem using 100 cells.

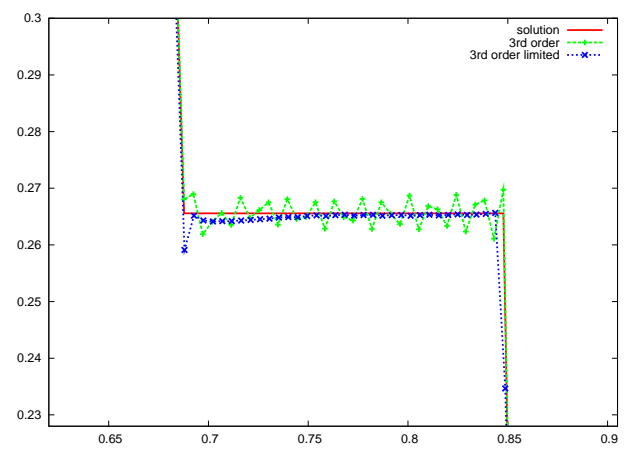

(b) Influence of the limitation at the shock front.

Figure 4: Numerical solutions for gas dynamics with and without limitation.

cells, around a mean state. Applying the above high-order limitation procedure, we obtain the limiting coefficients for the linearized Riemann invariants. Then, inverting the $3 \times 3$ linear system given by (20), we recover the limiting coefficients corresponding to the physical variables. Now, as displayed in Figure 4, if we perform our limitation on these quantities, we suppress most of the oscillations.

\subsection{Numerical results}

To demonstrate the accuracy and the robustness of our scheme on the gas dynamics system, we have run test cases taken from the literature. These results, displayed in Figure 5, have been obtained with our third-order scheme with slope limiters. In Figure 5(a), our scheme is perform on the Shu oscillating shock tube problem. Despite the strong perturbations, we note that the numerical solution is very close to the reference solution. In Figure 5(b), the run test case is the uniformly accelerated piston problem found in [9]. For a smooth solution this time, we notice once more, how accurate our solution is. Next, we compute the convergence rate of our DG scheme using a smooth solution of the gas dynamics equations. This solutions is constructed through the use of the Riemann invariants which write $J_{ \pm}=u \pm \frac{2}{\gamma-1} C$ for a gamma gas law. It is well known that the Riemann invariants are constant along the characteristic curves. These curves being defined by the differential 


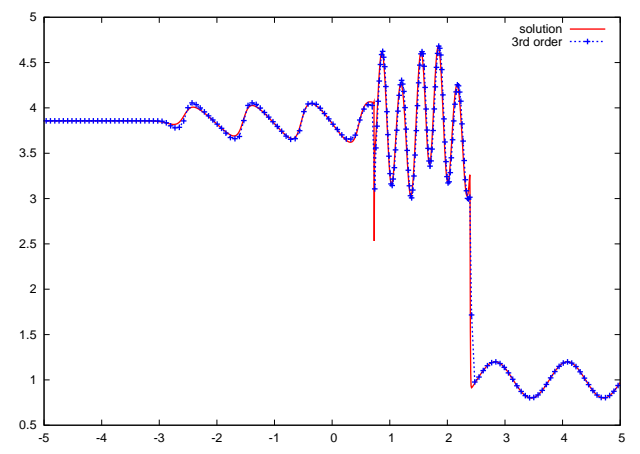

(a) Third-order DG for the Shu oscillating shock tube problem with 200 cells.

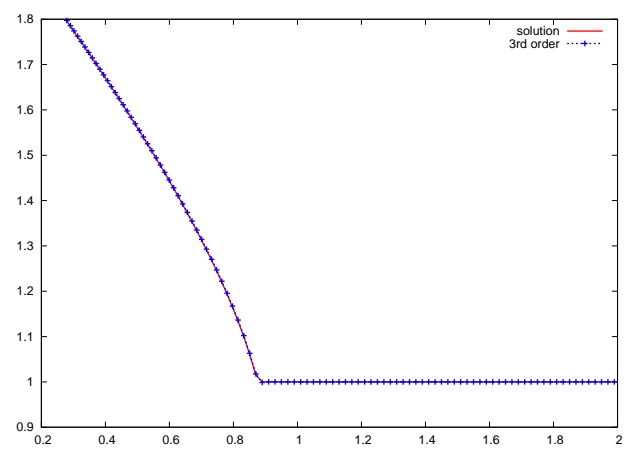

(b) Third-order DG for a uniformly accelerated piston problem taken from [9], with 100 cells

Figure 5: Numerical solutions for gas dynamics with limitation for third-order DG method.

equations

$$
\left(\mathcal{C}_{ \pm}\right) \frac{d X^{ \pm}}{d t}=u \pm C=\frac{(2 \pm(\gamma-1)) J_{+}+(2 \mp(\gamma-1)) J_{-}}{4}, \quad X(0)=x .
$$

Here, we have expressed the slopes of the characteristic curves in terms of the Riemann invariants. In the special case $\gamma=3$, we notice that the characteristic curves are straight lines. Hence, the gas dynamics equations are equivalent to two following Burgers equations

$$
\frac{\partial J_{ \pm}}{\partial t}+J_{ \pm} \frac{\partial J_{ \pm}}{\partial x}=0
$$

for which an analytical solution is easy to construct. Using this analytical solution we compute the global truncation error corresponding to our DG scheme and display it in Table 3.

\section{Conclusion}

We have presented a cell-centered DG discretization using Taylor basis for solving two-dimensional scalar conservations laws on general unstructured grids and also one-dimensional gas dynamics equations written in Lagrangian form. Numerical flux has been designed to enforce $L^{2}$ stability and an entropy inequality in the case of gas dynamics. A robust and accurate limitation procedure has been constructed. In future, we plan to investigate the 


\begin{tabular}{|c||c|c|c|}
\hline & & $L_{1}$ & $L_{2}$ \\
\hline \hline \multirow{4}{*}{ Gas dynamics } & first-order & 0.80 & 0.73 \\
\cline { 2 - 4 } & second-order & 2.25 & 2.26 \\
\cline { 2 - 4 } & second-order lim & 2.04 & 2.21 \\
\cline { 2 - 4 } & third-order & 3.39 & 3.15 \\
\cline { 2 - 4 } & third-order lim & 2.75 & 2.72 \\
\hline
\end{tabular}

Table 3: Rate of convergence for gas dynamics with and without the slope limitation for a smooth flow.

extension of the present DG discretization to two-dimensional Lagrangian hydrodynamics.

\section{References}

[1] R. Biswas, K. D. Devine and J.E. Flaherty, Parallel, adaptive finite element methods for conservation laws, Applied Numerical Mathematics 14:255-283, 1994.

[2] B. CockBurn, Discontinuous Galerkin methods School of Mathematics, University of Minnesota, 2003. Available from http://compmath.files.wordpress.com/2008/10/discontinuous_ galerkin_methods_cockburn.pdf.

[3] B. Cockburn, S.-Y. Lin and C.-W. Shu, TVB Runge-Kutta local projection discontinuous Galerkin finite element method for conservation laws II : one-dimensional, J. Comp. Phys. 84:90-113, 1989.

[4] B. Cockburn and C.-W. Shu, TVB Runge-Kutta local projection discontinuous Galerkin finite element method for conservation laws II : general framework, Math. Comput. 52:411-435, 1989.

[5] S. Hou And X.-D. LiU, Solutions of Multi-dimensional Hyperbolic Systems of Conservation Laws by Square Entropy Condition Satisfying Discontinuous Galerkin Method, Journal of Scientific Computing 31:127-151, 2007.

[6] G.-S. JiAng AND C.-W. Shu, On cell entropy inequality for discontinuous Galerkin methods, Math. Comput. 62:531-538, 1994. 
[7] D. Kuzmin, A vertex-based hierarchical slope limiter for p-adaptive discontinuous Galerkin methods, J. Comp. Appl. Math., 233:3077-3085, 2009.

[8] A. Kurganov, G. Petrova and B. Popov, Adaptive semi-discrete central-upwind schemes for non convex hyperbolic conservation laws, SIAM J. Sci. Comput., 29:2381-2401, 2007.

[9] P.-H. MAIRE, A high-order cell-centered Lagrangian scheme for twodimensional compressible fluid flows on unstructured meshes J. Comp. Phys. 228:2391-2425, 2009.

[10] C.-W. SHu, Discontinuous Galerkin methods: General approach and stability, S. Bertoluzza, S. Falletta, G. Russo and C.-W. Shu, Advanced Courses in Mathematics CRM Barcelona, Birkhäuser, Basel, 2009.

[11] M. YANG and Z.J. WANG, A parameter- free generalized moment limiter for high-order methods on unstructured grids Adv. Appl. Math. Mech. 1(4):451-480,2009. 